\title{
Lid nystagmus evoked by ocular convergence: an ocular electromyographic study
}

\author{
MICHAEL D. SANDERS, ${ }^{1}$ WILLIAM F. HOYT, ${ }^{2}$ \\ AND ROBERT B. DAROFF \\ From the Neuro-ophthalmology Service of the Departments of Neurology, Neurosurgery, and Ophthalmology \\ University of California School of Medicine, San Francisco, U.S.A.
}

In 1916 Pick described a nystagmus-like movement of the upper eyelids in a young woman with a slowly progressive neurological disease characterized by pseudobulbar and cerebellar signs. Her lids were motionless as she looked in the distance but, as she focused on an approaching object, they jerked up and down in rhythmic motions that increased as convergence increased. The lids also elevated in irregular step-like jerks as she followed an upward moving object. Gaze-evoked ocular nystagmus was present but Pick stressed that the lid 'nystagmus' was a separate motor phenomenon, related particularly to convergence.

There have been no further reports of this sign, which suggests that it is either extremely rare or difficult to recognize. The present report describes a case of lid nystagmus conforming to Pick's description and includes ocular electromyographic studies.

\section{CASE REPORT}

A 20-year-old man was admitted to the University of California Medical Center in San Francisco for the third time in April 1965 for signs and symptoms suggesting recurrence of his cerebellar sarcoma. He was initially referred to this hospital in January 1964 complaining of severe headaches, visual obscurations, and extreme nausea and vomiting. The latter had resulted in a 60-lb. weight loss during the previous six months. Examination demonstrated an ataxic, wide-based gait. There was gaze-evoked ocular nystagthus in all directions except downward. Visual acuity, optic discs and fundi were normal. Ventriculography showed a large 4th ventricular mass which on craniotomy was found to originate from the cerebellar vermis. The mass was excised and a rubber tube inserted through the aqueduct into the 3rd ventricle to facilitate drainage. Microscopic examination of the excised tissue showed it to be a cerebellar sarcoma. The patient improved following surgery and the nystag-

${ }^{1}$ Fellow in Neuro-ophthalmology sponsored by British Medical Research Council.

Reprint requests to Dr. Hoyt.

'USPHS Special Fellow in Neuro-ophthalmology, No. NB1820-01. mus diminished. He was discharged from the hospita距 after a course of radiotherapy had been commenced.

The patient's condition was stable for the next nine months, at which time vomiting again necessitated 0 readmission in October 1964. It was thought that these symptoms might be due to irritation from the drainage ${ }_{0}$ tube. Therefore a second craniotomy was performed and the tube removed. However tumour seeding was noted in the floor of the 4th ventricle. The vomiting ceased and the patient was discharged.

His final admission in April 1965 followed the development of severe headaches, diplopia, and paraesthesia on ${ }_{0}$ the left side of the face. A ventriculogram demonstrat $\mathrm{d}^{\infty}$ a left temporal lobe mass, probable implants in the rigtto lateral ventricle, and hydrocephalus. No further surgely was performed and the patient died at home in Juke 1965. Permission for necropsy was not granted. Theo ocular findings during this last admission constitute 通ec? subject of this report.

OPHTHALMiC EXAMINATION The pupils, visual acuity, 9 and fundus oculi remained normal. Ocular movements were full and conjugate, and there was no nystagmus in the primary position. Jerk nystagmus was evoked in all directions of gaze except downward. The most strikingo

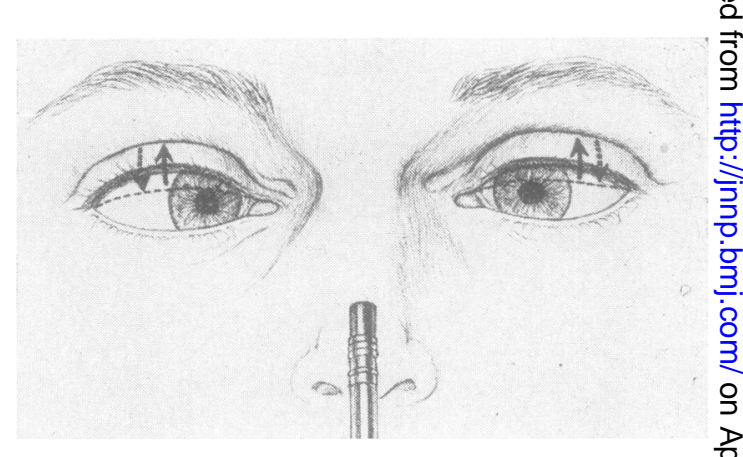

FIG. 1. Lid nystagmus evoked by convergence. The rapid phase of the lid movement is upward (solid N arrow). Lid movements are most pronounced during. marked convergence with the eyes slightly depressed 0 as indicated in diagram. 
finding occurred during convergence when rhythmic jerking movements appeared in both upper eyelids (Fig. 1); concurrent ocular nystagmus was not present. The amplitude of the lid nystagmus seemed proportional to the degree of convergence and the fast phase was always upward. The lid nystagmus was maximal when the resting lid position was at the upper pupillary margin and was absent on extreme elevation or depression. When the patient followed a vertically moving object, the lid movements were not smooth but appeared irregular and step-like.

ELECTROMYOGRAPHY Recordings were obtained from the left levator palpebrae superioris, paired alternately with the inferior rectus, the medial rectus, and the orbicularis oculi muscles, using fine concentric needle electrodes. Amplification was obtained with equipment that is standard for ocular electromyography (Loeffler, Slatt, and Hoyt, 1966).

Electrical activity in the levator palpebrae superioris and inferior oblique muscles was studied as the patient fixated upon an upward moving object held 14 in. from his face (Fig 2). When the eyes were turned downward, both muscles were electrically silent (inhibited). On upward gaze, as the inferior oblique muscle contracted (Fig 2), the electromyogram showed a pattern of phasic $4 / \mathrm{sec}$ bursts of motor units amid an interference pattern of increasing amplitude. The simultaneous recording from the contracting levator muscle showed at first brief and isolated low amplitude bursts of motor unit activity, then the progressive development of an interference pattern interrupted by rhythmic $(4 / \mathrm{sec})$ bursts of high voltage activity. The phasic discharges in both muscles were synchronous.

Electromyograms from the levator and medial rectus muscles were obtained during varying degrees of convergence and ocular elevation. With the eyes in the primary position the electrical pattern of tonus in the levator muscle was normal. As convergence began and increased, short bursts of high-amplitude spike discharges appeared on the levator tracing (Fig. 3). These intermittent bursts consisted of several motor units that appeared irregularly at a frequency of $2-3 / \mathrm{sec}$. The tracing from the medial rectus showed a normal interference pattern with no superimposed phasic activity. When the patient's limit of convergence was exceeded the eyes diverged and spike discharges in the levator decreased in frequency and displayed features of isolated unit discharges (Fig. 3). During steady convergence with the object 6 in. from the face and with the eyes moving upward from a depressed position, recordings showed regular 4/sec high-amplitude discharges superimposed upon a lower voltage interference pattern.

Recordings from a levator muscle and the orbicularis oculi muscle exhibited normal reciprocal innervation.

\section{DISCUSSION}

Lid nystagmus, a rhythmic upward jerking of the upper lids, is a rare abnormality of levator innervation. Several varieties have been recognized: (1) the normal coordinated movements of the lids and eyes during evoked or spontaneous vertical ocular nystagmus (Gowers, 1879; Bjørk and Kugelberg, 1953); (2) a synchronous jerking of the upper lids with the fast phase of lateral gaze-evoked nystagmus as described by Popper in 1916-this form of lid nystagmus is the subject of the accompanying report by Daroff, Hoyt, Sanders, and Nelson (1968); (3) a convergence-evoked lid nystagmus as described by Pick (1916).

Cerebellar ataxia and lid and ocular nystagmus

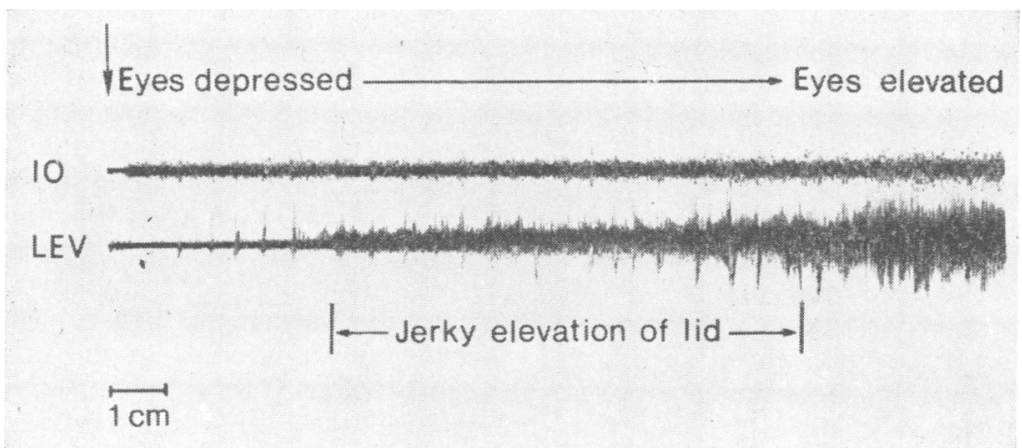

FIG. 2. Electromyograms from the levator palpebrae superioris (LEV) and the inferior oblique muscle (IO) recorded as the eyes and lids followed an upward moving object held $14 \mathrm{in}$. from the patient's face (gain $=100 \mu \mathrm{V} / \mathrm{cm}$ in upper tracing, and $200 \mu \mathrm{V} / \mathrm{cm}$ in the lower tracing; recording speed $=400 \mathrm{msec} / \mathrm{cm}$ ). The inferior oblique muscle showed an increasing interference pattern with some variable phasic low-voltage activity at $4 / \mathrm{sec}$. The smooth recruitment of motor units in the levator was interrupted by brief $4 / \mathrm{sec}$ high voltage bursts of activity. Clinically, the lids elevated in irregular step-like jerks. 


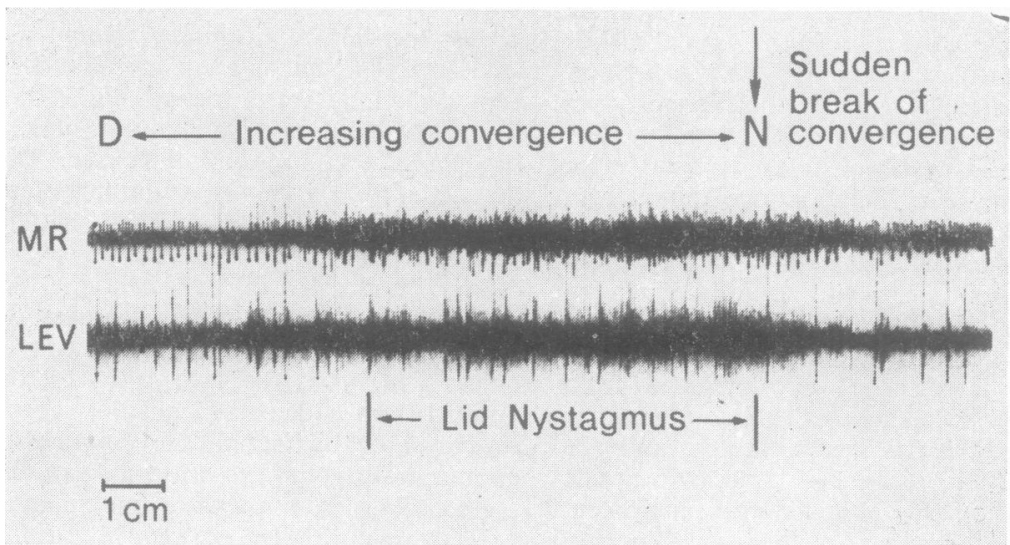

FIG. 3. Electromyograms from the levator palpebrae superioris (LEV) and the medial rectus muscle $(M R)$ during ocular convergence and evoked lid nystagmus (gain $=200 \mu \mathrm{V} / \mathrm{cm}$; recording speed $=400 \mathrm{msec} / \mathrm{cm})$. The patient's eyes were directed slightly below the horizontal plane. As convergence increased between $D$ (gaze in the distance) and $N$ (gaze at an object 6 in. from the nose) and as a normal interference pattern appeared in the medial rectus muscle, single high-voltage units in the levator palpebrae superioris began to appear in phasic 3-4/sec bursts superimposed upon an interference pattern of increasing amplitude. Upward jerking nystagmus of the upper lid appeared as convergence approached $N$. The high-voltage bursts and the amplitude of the interference pattern in the levator palpebrae superioris decreased abruptly at the sudden break of convergence.

were prominent features in both Pick's case and the case reported here. However, in neither case was the clinical data sufficient to allow precise localization of the brain-stem lesion.

Pick postulated that the lid activity reflected abnormal spread of excitation among cell groups within the oculomotor nuclei. The electromyographic studies of the levator and extraocular muscles in our patient demonstrate a pattern of abnormal levator innervation which contributes to our understanding of the mechanism of this form of lid nystagmus.

The levator electromyograms showed normal recruitment of low voltage (tonic) units, and a normal inhibitory pattern in downward gaze and blinking. The most striking electrophysiological feature was the abnormal facilitation of single or multiple high-voltage discharges which appeared on convergence. At the onset of convergence single high-voltage units appeared in the EMG at a frequency of $2-3 / \mathrm{sec}$. As convergence increased there was a progressive build-up of additional high-voltage units in the form of bursts, and also an increase in the normal interference pattern. Clinically the lids showed an upward jerking nystagmoid movement with an amplitude of 1-2 mm and a rate of $2-3 / \mathrm{sec}$. When the patient followed an upward moving object in the distance the lid movements were smooth and there were only sporadic high-voltage units the electromyogram. However when the same moveco ment was performed with the eyes converged, lar of isolated units discharged irregularly at 4-6/seg. these increased in amplitude and frequency as the lid progressively elevated. The following movement of the lids became irregular and step-like when the eyes were converged upon a point $14 \mathrm{in}$. away but at no time was there any visible lid nystagmus. At the limit of upward gaze the bursts of electrical activity in the levator were of very high amplitude and were $\stackrel{\Phi}{\varrho}$ synchronous with the low-amplitude discharges in $\overrightarrow{\vec{O}}$ the contracting inferior oblique muscle.

The abnormal facilitation of the high-voltage units from the levator palpebrae superioris muscle occurred principally in convergence. Lid nystagmus was produced by these high-voltage bursts only when the eyes converged in the horizontal plane or slightly below. It therefore appears from the data in our case that lid nystagmus becomes visible only 8 when certain criteria are met: (1) the frequency of $₹$ phasic innervation must not exceed 2-3/sec; if the 0 frequency is greater the lid will not descend sufficiently between jerks to permit phasic movement; $\frac{D}{2}$ (2) the lid must be in a partially relaxed position; if it is too high, upward jerking cannot occur and, if $N$ too low, levator inhibition diminishes phasic $N$ facilitation; (3) each abnormal innervational burst ్్ㅇ 
must contain a sufficient number of high-voltage units to produce a twitch response in the levator, resulting in an upward jerk of the lid.

Levator tonus normally increases with visual attention and convergence, resulting in a widening of the palpebral fissures (Kennard and Glaser, 1964). However the high-voltage units recorded in our patient are not normally present and their origin is unexplained.

Other abnormalities of levator innervation occur in diseases of the rostral midbrain and the basal ganglia. The pathological lid retraction (Collier, 1927) attributed to lesions in the region of the posterior commissure exemplifies abnormal facilitation of levator tonus, due presumably to disruption of the pretectal inhibitory influence on the levator motoneurones. We are unaware of any electromyographic studies of the levator muscle in this condition but the phasic levator activity in our patient on elevation (without associated visible lid nystagmus) suggests that Collier's sign may represent a similar innervational disorder. The irregular lid movements seen when our patient followed a vertically moving object also occur in Parkinson's disease and related extrapyramidal disorders. These movements represent sporadic high-voltage levator discharges which do not occur in the pattern necessary to produce lid nystagmus.

In an accompanying report (Daroff et al., 1968) we describe a distinctly different type of lid nystagmus, which was gaze-evoked and in synchrony with an associated ocular nystagmus. The lid movements, in contrast to the present case, were inhibited by convergence and caused by a focal lesion of the lateral medulla.

\section{SUMMARY}

Rhythmic jerking of the lids at the rate of 2-3 beats/sec may be provoked exclusively by convergence with the eyes in the horizontal plane. This rare sign (Pick's lid nystagmus), exemplified in a report of a patient with a cerebellar sarcoma, is the visible expression of a peculiar innervational anomaly of levator motoneurons. Electromyographic studies of the levator palpebrae superioris showed high-voltage phasic bursts of motor units that occurred during convergence of the eyes.

\section{REFERENCES}

Bjørk, A., and Kugelberg, E. (1953). The electrical activity of the muscles of the eye and eyelids in various positions and during movement. Electroenceph. clin. Neurophysiol., 5, 595-602.

Collier, J. (1927). Nuclear ophthalmoplegia, with especial reference to retraction of the lids and ptosis and to lesions of the posterior commissure. Brain., 50, 488-498.

Daroff, R. B., Hoyt, W. F., Sanders, M. D., and Nelson, L. (1968). Gaze-evoked eyelid and ocular nystagmus inhibited by the near reflex: Unusual ocular motor phenomena in a lateral medullary syndrome. J. Neurol. Neurosurg. Psychiat., 31, 362-367.

Gowers, W. R. (1879). The movements of the eyelids. Med.-chir. Trans., 62, 429-440.

Kennard, D. W., and Glaser, G. H. (1964). An analysis of eyelid movements. J. nerv. ment. Dis., 139, 31-48.

Loeffler, J. D., Slatt, B., and Hoyt, W. F. (1966). Motor abnormalities of the eyelids in Parkinson's disease. Electromyographic observations. Arch. Ophthal., 76, 178-185.

Pick, A. (1916). Kleine Beiträge zur Neurologie des Auges, 11: Uber den Nystagmus der Bulbi begleitende gleichartige Bewegungen des oberen Augenlides (Nystagmus des Oberlides). Arch. Augenheilk., 80, 36-40.

Popper, E. (1916). Ein Beitrag zur Frage des 'Lidnystagmus'. Mschr. Psychiat. Neurol., 39, 188-192. 